\title{
Erweitertes Zusammenwirken in der Landwirtschaft - zur Diskussion
}

\author{
Lars Wolf
}

\section{Zusammenfassung}

In der Landwirtschaft wurde schon immer Technik eingesetzt, auch wenn aus heutiger Sicht viele Verfahren sehr einfach und kaum technisiert erscheinen mögen. Insbesondere in jüngerer Zeit kamen verschiedene Arten von Beobachtungs- und Prognoseverfahren hinzu, welche nun Informationen über den Zustand von Tieren und Pflanzen liefern können. Durch die informationstechnischen Entwicklungen der letzten Jahre ergeben sich somit vielfältige Fragestellungen bezüglich des Zusammenwirkens von Menschen, Tieren und Pflanzen: Welche Möglichkeiten ergeben sich durch die Digitalisierung, beispielsweise durch den Einsatz von Sensorik und die Einführung von Verfahren aus der künstlichen Intelligenz? Wie kann ein Zusammenwirken von natürlicher und künstlicher Intelligenz in der Landwirtschaft aussehen und welche Auswirkungen, welcher Art und auf was bezogen, kann es hierdurch geben?

Die Ausarbeitung basiert auf einem Vortrag, den der Verfasser gemeinsam mit Engel Hessel am 15.2.2019 auf dem 1. BWG-Symposium über das Zusammenwirken von natürlicher und künstlicher Intelligenz gehalten hatte.

\footnotetext{
L. Wolf $(\bowtie)$

Institut für Betriebssysteme und Rechnerverbund der TU Braunschweig, Braunschweig, Deutschland

E-Mail: wolf@ibr.cs.tu-bs.de

(C) The Author(s) 2021

R. Haux et al. (Hrsg.), Zusammenwirken von natürlicher und künstlicher 


\section{Schlüsselwörter}

Digitale Landwirtschaft · Smart Farming · Precision Farming · Precision

Livestock Farming · Wireless Sensor Networks · Internet of Things ·

Künstliche Intelligenz

\section{$1 \quad$ Einleitung}

Schon seit jeher in der Menschheitsgeschichte gibt es ein Zusammenwirken von Menschen, Tieren und Pflanzen. Insbesondere seit Beginn der Landwirtschaft vor einigen Tausend Jahren hat sich dies durch Ackerbau und Viehhaltung sowie entsprechende Nutzungskreisläufe in vielfältiger Weise verstärkt. Technik wurde schon immer in der Landwirtschaft eingesetzt, auch wenn aus heutiger Sicht viele Verfahren sehr einfach und kaum technisiert erscheinen mögen. Über die Zeit sind viele technische, mechanische Komponenten, zum Beispiel für die Bodenbearbeitung, eingeführt worden. Doch auch alle Möglichkeiten zum Erhalt von Informationen über den Zustand von Tieren und Pflanzen sind von sehr großer Bedeutung. In früheren Zeiten gab es hierfür aber meist keine technische Unterstützung, was sich in jüngerer Zeit durch verschiedene Arten von Beobachtungsund Prognoseverfahren substanziell geändert hat und zu neuen Möglichkeiten führt, weil diese Methoden nun Informationen über den Zustand von Tieren und Pflanzen liefern können.

Vor allem durch die informationstechnischen Entwicklungen der letzten Jahre ergeben sich zusätzliche und neuartige Fragestellungen bezüglich des Zusammenwirkens von Menschen, Tieren und Pflanzen: Welche Möglichkeiten ergeben sich durch die Digitalisierung, beispielsweise durch den Einsatz von Sensorik und die Einführung von Verfahren aus der künstlichen Intelligenz? Wie kann ein Zusammenwirken von natürlicher und künstlicher Intelligenz in der Landwirtschaft aussehen und welche Auswirkungen, welcher Art und auf was bezogen, kann es hierdurch geben?

Bei einem solchen erweiterten Zusammenwirken von Menschen, Tieren, Pflanzen und (intelligenten) Maschinen, also verschiedenartige lebende und nicht lebende Entitäten, bestehen diverse Abhängigkeiten und wechselseitige Beeinflussungen, die mit betrachtet werden müssen. Dabei ist zu beachten, dass in der Landwirtschaft der jeweilige Anwendungskontext für den Einsatz von Technik immer wichtig ist. Zudem müssen verschiedene Herausforderungen angegangen werden. So sind Tiere und Pflanzen recht individuell und haben unterschiedliche Eigenschaften und Umgebungsbedingungen, dies 
gilt auch für mehrere Individuen einer Art. Typischerweise ist auf einen sparsamen Umgang mit Ressourcen zu achten, und dies nicht nur kurzfristig, sondern auf lange Zeiträume. Des Weiteren muss die eingesetzte Technik robust sein, sodass beispielsweise ausgebrachte Sensorik und Aktorik auch unter widrigen Einsatzbedingungen (auf dem Acker, im Stall, bei stark schwankenden Wetterbedingungen, bei deutlicher Verschmutzung etc.) längere Zeit eigenständig und zuverlässig arbeiten kann sowie auch bei sich verändernden Umgebungseigenschaften weiterhin noch möglichst nützliche Resultate liefert.

Als essenzielle Herausforderung an informationstechnische Systeme bezüglich des erweiterten Zusammenwirkens von Menschen, Tieren und Pflanzen stellt sich dann auch die Frage nach der Verlässlichkeit von Algorithmen und Systemen: Wenn Algorithmen Empfehlungen geben oder gar Entscheidungen treffen, beispielsweise bezüglich Krankheiten oder Reifezustand, was sind prinzipielle Auswirkungen auf alle direkt und auch indirekt Beteiligte?

Im folgenden Abschnitt werden zunächst einige derzeitige technische Möglichkeiten im Pflanzenbau vorgestellt. Anschließend werden die im letzten Absatz angesprochenen grundsätzlichen Fragen des Zusammenwirkens, aber auch der Auswirkungen, erneut aufgegriffen. Der vorliegende Text soll vor allem zu Gedanken sowie Diskussionen anregen; es geht weniger darum Lösungen darzustellen.

\section{Einsatz von Sensorik im Pflanzenbau}

Erfolgreiche Landwirtschaft braucht eine Vielzahl an Informationen, die zudem möglichst aktuell sein sollten. Hierzu gehören Informationen über Umweltaspekte wie aktuelle und kommende Wetterbedingungen und über den Zustand von Kulturpflanzen, andere Pflanzen, den Boden etc. - in Bezug auf verschiedene Parameter wie unter anderem Temperatur, Niederschlag, Luftfeuchtigkeit, Bodenfeuchte, Wind, Sonnenschein, Lichtintensität. (Fraunhofer 2014).

Solche Informationen, zum Beispiel über den Zustand von Pflanzen, können auf unterschiedliche Weise gewonnen werden, siehe u. a. (Link-Dolezal et al. 2012). In technischer Form, also nicht nur durch menschliche Beobachtung, können hierzu zum einen stationäre Sensoren auf oder gar in dem Ackerboden, dem Gewächshaus etc. eingesetzt werden (wie es in Abb. 1 (links) zu sehen ist). Zum anderen kann die Informationserfassung aber auch durch mobile Geräte oder Fernerkundung erfolgen: durch Sensoren an Landmaschinen wie Traktoren, siehe beispielsweise (Gernert et al. 2019) und Abb. 1 (rechts), oder anderen mobilen Arbeitsmaschinen während der Bearbeitung, was gegebenenfalls auch eine 

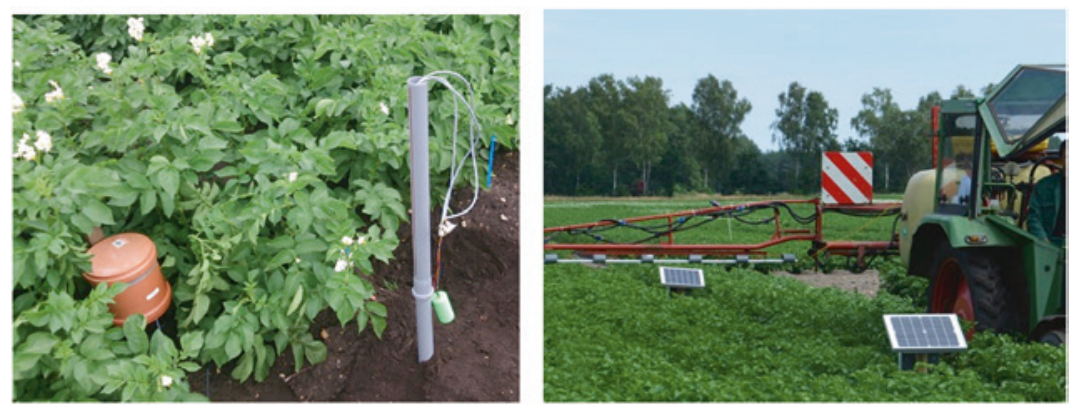

Abb. 1 Sensorik zwischen Kartoffelpflanzen sowie Landmaschine mit Technik zur Übernahme von Messwerten. (Quelle: Eigene Photographien)

direkte Beeinflussung der Aktorik ermöglichen kann, oder auch durch Erfassung mittels Satelliten, Flugzeugen oder UAVs (Unmanned Aerial Vehicle).

Mit Hilfe von solchermaßen detailliert und möglichst teilflächenspezifisch erfassten Informationen können dann in Kombination mit langfristig nachgeführten Angaben zu Boden- und Klimaparametern möglichst gute Entscheidungen für die konkrete Bewirtschaftung von Flächen getroffen werden und dies unter Berücksichtigung der natürlichen sowie der betrieblichen Ressourcen. Statt nur räumlich oder zeitlich beschränkt verfügbarer Informationen, wie zum Beispiel im Falle von regionalen Wetterstationen, könnten somit feingranular aufgelöste, mikroklimatische Bedingungen einzelner Teilflächen in Prognosemodelle eingehen.

Durch neuartige Technik können sich aber auch ganz neue Möglichkeiten zur Feldbearbeitung ergeben. Statt dem bisherigen Trend in der Landtechnik zu immer größeren Arbeitsmaschinen, könnten kleinere, autonome Feldroboter eingesetzt werden (Gaus et al. 2017). Diese Roboter könnten dann auch statt des derzeitigen Ansatzes einer großflächigen Bearbeitung und Behandlung von vielen Pflanzen, hin zu einer kleinteiligen, gegebenenfalls individuellen Behandlung von Pflanzen führen, sodass beispielsweise nur an den notwendigen Stellen gedüngt oder unerwünschte Pflanzen entfernt werden. Hierzu muss der Roboter die jeweilige Situation eigenständig erkennen, analysieren, geeignete Entscheidungen fällen und diese dann umsetzen.

Nicht nur auf dem Feld, sondern auch in späteren Phasen wie dem Transport und der Lagerung des Ernteguts kann der Einsatz sensorbasierter Verfahren 
sehr nützlich sein. So ist die Verfügbarkeit guter Lagerungsverfahren für landwirtschaftliche Produkte von hoher Bedeutung. Beispielsweise bezogen auf die Kartoffelproduktion werden Lebensmittelverluste auf der Erzeugerstufe überwiegend durch Lagerverluste verursacht (Peter et al. 2013). Durch geeignete Nutzung von Sensorik direkt zwischen den gelagerten Produkten wie Kartoffeln, könnten die Lagerbedingungen (Temperatur, Luftfeuchte) optimiert werden, sodass Verluste reduziert werden können bei gleichzeitiger Verringerung des Ressourcenbedarfs zur Klimatisierung während der Lagerung.

Allerdings ergeben sich je nach eingesetzter Technik vielfältige Herausforderungen. Neben generellen Fragen, die beim Einsatz von verschiedenen Technologien auftreten und insbesondere im Bereich der Informationstechnologie immer wieder kritisch sind, wie sie die Interoperabilität zwischen Geräten verschiedener Hersteller und den von diesen gelieferten Daten, passende Schnittstellen etc., darstellen, ist zum Beispiel im Umfeld des Einsatzes von Sensornetzen daran zu forschen, wie Robustheit und Verfügbarkeit erreicht werden können (Hartung et al. 2017). Diese Eigenschaften müssen auch bei rauen Umgebungsbedingungen, zu niedrigen Kosten und bei beschränkten Energieressourcen (wie möglichst kleinen Batterien) bereitgestellt werden. Da das Einsatzgebiet im ländlichen Raum liegt, ist noch mehr als im urbanen Umfeld, eine Frage, wie die generierten Daten übertragen werden können, sodass sie beispielsweise auf dem Hof des Landwirts für die Auswertung und Entscheidungsfindung nutzbar sind. So muss bei der Gestaltung von Technologien berücksichtigt werden, dass im ruralen Raum typischerweise nur limitierte Konnektivität und begrenzte Datenübertragungsmöglichkeiten vorhanden sind. Auch wenn sich die Verfügbarkeit von mobilen, drahtlosen Netzen zukünftig auch auf dem Land verbessern wird, durch Mobilfunktechniken wie 4G/LTE, 5G oder auch Satellitenbasierten Netzen wie OneWeb, bleibt es aufgrund von Kostengesichtspunkten wichtig zu überlegen, welche Ansätze zur Datenübertragung genutzt werden.

\section{$3 \quad$ Auswirkungen \& Bedeutung}

Was bedeutet nun die Verfügbarkeit von vielfältigen neuen Techniken bezüglich des Zusammenwirkens von Menschen, Tieren und Pflanzen in der Landwirtschaft? 
Es ergeben sich vielfältige Herausforderungen bereits aus dem, auch als „Big Data" bezeichneten, außerordentlich umfangreichen Sammeln, Verarbeiten und Verwenden an Daten. Zum einen gibt es selbstverständlich eine Reihe an eher technischen Aspekten, zum Beispiel:

- Wie einfach können Daten erhoben werden?

- Wie einfach kann auf diese Daten zugegriffen werden?

- Wie einfach können sie verarbeitet werden?

Hierzu verwandte Überlegungen lassen sich ableiten, die dann aber nicht funktionale Aspekte betreffen. Hierzu gehören dann unter anderem Fragen wie:

- Wie sicher sind Daten \& Services?

- Welche Privacy-Aspekte sind wann und wie zu berücksichtigen?

Darüber hinaus gibt es aber eine Vielzahl weiterer Aspekte, die zum Teil juristischer Natur sind, vor allem aber auch wirtschaftliche Gesichtspunkte mit einbringen. Auch wenn es zu einigen aus den entsprechenden Disziplinen Antworten gibt, so ist dennoch generell eine Erörterung sinnvoll. $\mathrm{Zu}$ diesen Gedanken gehören beispielsweise:

- Wer darf Daten an welchen Stellen aufnehmen? Darf beispielsweise von beliebigen Unternehmen per Satellit eine Kartographierung und Einordnung der Bodengüte eines Ackers vorgenommen werden?

- Wem gehören die Daten und wem sollten sie gehören? Demjenigen, der die (Roh)Daten erfasst hat, d. h. möglicherweise ein Unternehmen, welches im Auftrag eines Landwirts einen Bearbeitungsschritt wie die Getreideernte durchgeführt hat? Oder gehören sie dem Eigentümer des Bodens? Wie ist die Situation, wenn der Acker verpachtet ist?

- Wer profitiert von den Daten und wer sollte hiervon einen Nutzen haben können? Wie in anderen Fällen kann auch hier noch eine weitere Unterscheidung erfolgen: Wer profitiert von den Rohdaten und, vor allem, wer profitiert von den Analyseergebnissen? Auch hier gibt es, wie bei der Frage nach dem Eigentum an Daten, verschiedene Beteiligte.

Zudem gibt es viele weitere, durchaus essenzielle Fragen danach, wie sich die Verfügbarkeit von umfangreichen, extern erfassten Kenndaten und Informationen 
auf das Selbstverständnis der beteiligten Personen, insbesondere die Landwirte auswirkt. Bislang wurden durch diese aufgrund ihrer langjährigen Erfahrungen Entscheidungen bezüglich der Bewirtschaftung von Flächen, oder ähnliches für andere landwirtschaftliche Aufgaben, getroffen. Wie sieht dies zukünftig aus, sind diese Erfahrungen nun nicht mehr im gleichen Maße wie zuvor nötig oder wertvoll? Welche Rolle sollte der Mensch und speziell der Landwirt hierbei spielen?

Auf Tierhaltung bezogen gibt es gleichermaßen wichtige Fragen. Durch verschiedenste Arten von technischen Systemen kann das Verhalten und der Zustand von Nutztieren erfasst werden. Hieraus lassen sich dann mit Entscheidungsunterstützungssystemen durch Menschen oder auch direkt nur von Algorithmen, ohne explizite menschliche Mitwirkung, Schlussfolgerungen ziehen, wie mit welchen Tieren umgegangen werden soll. Derartige Schlussfolgerungen können dann auch auf Entscheidungen hinauslaufen, beispielsweise welches Tier eine bestimmte Behandlung bekommen wird, welches weiter gefüttert und welches geschlachtet wird. Technisch könnten dann möglicherweise auch vollautomatische Stall-/ Schlachthof-/Verarbeitungszentren aufgebaut werden, wo Menschen kaum noch eine reguläre Rolle spielen, sondern alle anfallenden Arbeiten von Maschinen und Robotern sowie der dazugehörigen intelligenten Software übernommen werden. Auch eine gezielte Verhaltensbeeinflussung bei Tieren durch Brain-ComputerInterfaces könnte zukünftig möglich sein, sodass rechnerbasiert Befehle wie gehe zum Futtertrog oder schlussendlich auch gehe zum Schlachtpunkt an die Nutztiere erfolgen könnten. Wären solche Tierprodukt-Erzeugungszentren aus ethischer Sicht akzeptabel?

Dies bedeutet, dass sich vielfältige Gedanken über Autonomie und Verantwortung ableiten lassen, beispielsweise:

- Was bedeutet der Verlust an (landwirtschaftlicher) Kompetenz beim Menschen?

- Wie wichtig sind von Menschen getroffene Entscheidungen?

- Ist es akzeptabel, wenn ein Algorithmus entscheidet, wann ein Tier gemolken, gefüttert, ... wird und letztendlich auch welches Tier geschlachtet wird?

- Wären vollautomatische Stall-/Schlachthof-/Verarbeitungszentren akzeptabel?

- Welche rechtliche und moralische Verantwortlichkeit ergibt sich?

Schließlich stellt sich für unsere Gesellschaft die Frage, wie wir zukünftig unsere Lebensmittel produzieren und welches Essen wir als Menschen verzehren wollen. 


\section{Literatur}

Fraunhofer-Institut für Experimentelles Software Engineering (2014). Sensoreinsatz und Datenanalyse in der Landwirtschaft. In digitale welt (2014), November (S. 28-31).

Gaus, C.-C., Minßen, T. -F., Urso, L.-M., de Witte, T., \& Wegener, J. (2017). Mit autonomen Landmaschinen zu neuen Pflanzenbausystemen. Projektbericht. www.orgprints. org/32437. Zugegriffen: 26. Apr. 2019.

Gernert, B., Schlichter J., \& Wolf, L. (2019). PotatoScanner - A mobile delay tolerant wireless sensor node for smart farming applications. In Proceedings of the 15th IEEE international conference on distributed computing in sensor systems, DCOSS 2019, Santorini Island, Greece.

Hartung, R., Kulau, U., Gernert, B., Rottmann, S., \& Wolf, L. (2017). On the experiences with testbeds and applications in precision farming. In Proceedings of the first ACM international workshop on the engineering of reliable, robust, and secure embedded wireless sensing systems, FAILSAFE 2017, Delft, The Netherlands.

Link-Dolezal, J., Zecha, C., \& Claupein, W. (2012). Sensoreinsatz und Datenanalyse in der Landwirtschaft. In 16. GMA/ITG-Fachtagung Sensoren und Messsysteme.

Peter, G., Kuhnert, H., Haß, M., Banse, M., Roser, S., Trierweiler, B., \& Adler, C. (2013). Einschätzung der pflanzlichen Lebensmittelverluste im Bereich der landwirtschaftlichen Urproduktion. In Johann Heinrich von Thünen-Institut, Max-Rubner-Institut, Julius Kühn-Institut.

Open Access Dieses Kapitel wird unter der Creative Commons Namensnennung 4.0 International Lizenz (http://creativecommons.org/licenses/by/4.0/deed.de) veröffentlicht, welche die Nutzung, Vervielfältigung, Bearbeitung, Verbreitung und Wiedergabe in jeglichem Medium und Format erlaubt, sofern Sie den/die ursprünglichen Autor(en) und die Quelle ordnungsgemäß nennen, einen Link zur Creative Commons Lizenz beifügen und angeben, ob Änderungen vorgenommen wurden.

Die in diesem Kapitel enthaltenen Bilder und sonstiges Drittmaterial unterliegen ebenfalls der genannten Creative Commons Lizenz, sofern sich aus der Abbildungslegende nichts anderes ergibt. Sofern das betreffende Material nicht unter der genannten Creative Commons Lizenz steht und die betreffende Handlung nicht nach gesetzlichen Vorschriften erlaubt ist, ist für die oben aufgeführten Weiterverwendungen des Materials die Einwilligung des jeweiligen Rechteinhabers einzuholen.

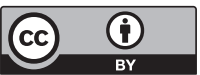

